\title{
Influence of Kerr nonlinearity on PT- transition in coupled fibre lasers
}

Sergey V. Smirnov, Maxim O. Makarenko, Andrey A. Sukhorukov, llya D. Vatnik, Dmitry V. Churkin

Sergey V. Smirnov, Maxim O. Makarenko, Andrey A. Sukhorukov, llya D. Vatnik, Dmitry V. Churkin, "Influence of Kerr nonlinearity on PT-transition in coupled fibre lasers," Proc. SPIE 10683, Fiber Lasers and Glass Photonics: Materials through Applications, 106832I (17 May 2018); doi: $10.1117 / 12.2306118$

SPIE. Event: SPIE Photonics Europe, 2018, Strasbourg, France 


\title{
Influence of Kerr nonlinearity on PT-transition in coupled fibre lasers
}

\author{
Sergey V. Smirnov*a, Maxim O. Makarenko ${ }^{\mathrm{a}}$, \\ Andrey A. Sukhorukov ${ }^{b}$, Ilya D. Vatnik ${ }^{\mathrm{a}}$, Dmitry V. Churkin ${ }^{\mathrm{a}}$ \\ ${ }^{a}$ Novosibirsk State University, Pirogova St. 2, Novosibirsk, 630090, Russia; \\ ${ }^{\mathrm{b}}$ Nonlinear Physics Centre, Research School of Physics and Engineering, \\ The Australian National University, Canberra ACT 0200, Australia.
}

\begin{abstract}
This work investigates a concept of coupled fiber lasers exhibiting PT-symmetry and a PT-transition between PTsymmetric and PT-broken lasing states. We consider a system operated via Raman gain comprising two fiber loops (ring cavities) connected to each other by means of two fiber couplers with adjustable phase shift between them. By changing the phase shift or/and amplification (loss) in fiber loops, one can switch between generation regimes, realizing either PTsymmetric or PT-broken solution. In the PT-symmetric lasing regime, equal powers are generated in both cavities despite only active one is pumped. We make theoretical and numerical description of the proposed coupled fiber lasers starting with the simple discrete matrix model taking into account coupling, phase delays, gain (which is assumed to be saturated), losses and nonlinear phase shift. We show how the PT-transition is affected by self-phase modulation inside the fiber cavity and investigate requirements that should be met in order to observe PT-transition experimentally despite Kerr effect that violates exact symmetry conditions. In particular, we show that PT-transition may be observable only near lasing threshold. Further on we adopt more sophisticated model based on Nonlinear Schrödinger equation for PT fiber laser. Taking into account quasi-CW polychromatic radiation with typical spectral bandwidth of fiber Raman lasers, chromatic dispersion and Kerr nonlinearity, we demonstrate both PT-symmetric and PT-broken lasing in a fiber laser.
\end{abstract}

Keywords: fibre laser, PT symmetry.

\section{INTRODUCTION}

The concept of parity-time symmetry in optics attracts great deal of attention. Recently, a parity-time symmetry-breaking microring laser has been experimentally demonstrated ${ }^{1,2}$. In particular, the latter paper demonstrates a single-mode operation in an initially multi-mode system what was achieved by means of stronger mode discrimination close to a PTtransition point. Further on, a single transverse mode operation in a system of coupled microring lasers was demonstrated near the exceptional point ${ }^{3}$. In a pair of coupled microdisk quantum cascade lasers, the reversal of generated power dependence was identified in the vicinity of exceptional points ${ }^{4}$. A realization of the PT symmetry-based mode-locking has been theoretically proposed ${ }^{5}$, and PT fiber cavity laser was developed ${ }^{6}$. Although most papers consider microcavities as a test bed to study PT transition, recently we propose a concept of coupled fiber lasers exhibiting PTsymmetry properties ${ }^{7}$. In this work, we study the proposed concept in more details. In particular, we make theoretical and numerical description of the proposed fiber system starting with the simple discrete stationary matrix model taking into account coupling, phase delays, saturable gain and losses. Further we take into account nonlinear phase shift caused by Kerr nonlinearity of optical fibers. We show how the PT-transition is affected by self-phase modulation inside the fiber cavity. We also discuss PT-transition in a more sophisticated model based on Nonlinear Schrödinger equation which takes into account fast temporal dynamics and evolution of optical spectrum of laser radiation.

\section{LASER LAYOUT}

The scheme of PT-symmetric fiber system ${ }^{7}$ is shown in Fig. 1. The scheme comprises two optical fiber loops, one with gain $\Gamma_{1}$, another one with loss (optical attenuator) $\Gamma_{2}$, connected to each other by means of a pair of optical couplers with phase shift between them. Varying phase shifts $\varphi_{1}, \varphi_{2}$, one can change coupling ratio between two fiber loops.

*smirnov@lab.nsu.ru; phone +7 383363 4165, fax +7 383363 4265; www.nsu.ru

Fiber Lasers and Glass Photonics: Materials through Applications, edited by Stefano Taccheo, Jacob I. Mackenzie, Maurizio Ferrari, Proc. of SPIE Vol. 10683 106832 I · 2018 SPIE · CCC code: 0277-786X/18/\$18 · doi: 10.1117/12.2306118 
Thus, zero relative phase shift $\delta \varphi=\varphi_{1}-\varphi_{2}=0$ corresponds to the case of decoupled fiber loops. In contrast, when $\delta \varphi=\pi$, the loops are fully coupled so that $100 \%$ of radiation exiting the first loop enters the second one and vice versa. When the coupling ratio between loops are strong enough, PT-symmetric lasing can be achieved what means symmetrical power distribution between two fiber loops. In contrast, when coupling ratio is weak, PT-broken lasing regime is realized what means that power inside amplifying fiber loop is higher than power inside attenuator loop ${ }^{7}$. Now we'll study in more details how the PT transition is affected by Kerr nonlinearity of intra-cavity optical fibers.

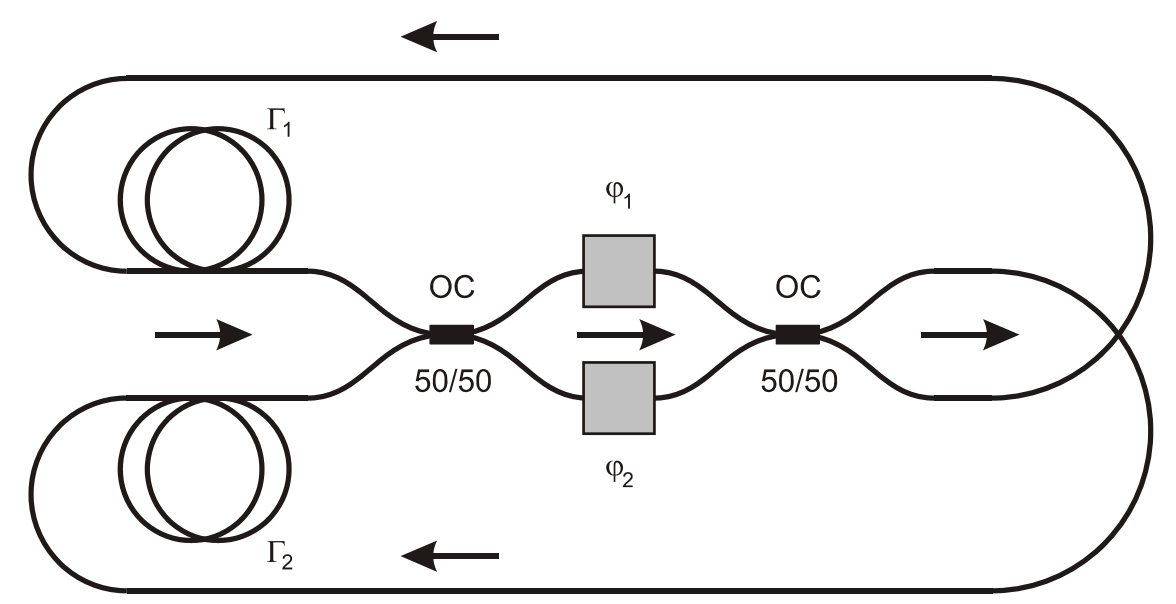

Fig. 1. Scheme of PT-symmetrical fiber laser under consideration. $\Gamma_{1}$ and $\Gamma_{2}-$ optical gain and loss, $\varphi_{1}$ and $\varphi_{2}-$ variable phase shifters, OC $-50 / 50$ optical couplers. The arrows indicate light propagation directions. Amplifying optical fiber $\Gamma_{1}$ requires optical pump module (not shown in this figure.)

\section{STATIONARY MATRIX MODEL}

In order to describe lasing properties of PT-symmetric fiber laser system under consideration, let's start with a quite simple matrix model. Let $\left(u_{1}, u_{2}\right)$ be a vector of radiation amplitudes, where $u_{1}$ and $u_{2}$ are radiation amplitudes inside gain and loss fiber loops respectively. Evolution of this vector can be described by means of $2 \times 2$ matrix being product of $2 \times 2$ matrices of separate optical fiber elements shown in Fig. 1. Matching of fiber optical elements and matrices is as follows:

$$
A_{O C}=\frac{1}{\sqrt{2}}\left(\begin{array}{ll}
1 & i \\
i & 1
\end{array}\right), \quad A_{\varphi}=\left(\begin{array}{cc}
\exp \left(i \varphi_{1}\right) & 1 \\
1 & \exp \left(i \varphi_{2}\right)
\end{array}\right), \quad A_{C C}=\left(\begin{array}{ll}
0 & 1 \\
1 & 0
\end{array}\right), \quad A_{\Gamma}=\left(\begin{array}{cc}
\Gamma_{1} e^{i \gamma\left|u_{1}\right|^{2}} & 0 \\
0 & \Gamma_{2}
\end{array}\right),
$$

where $A_{\mathrm{OC}}$ stands for optical coupler matrix, $A_{\varphi}$ describes phase rotator, $A_{\mathrm{CC}}$ stands for matrix of optical cross-connection and $A_{\Gamma}$ is matrix of optical amplification and losses with saturable gain $\Gamma_{1}$ :

$$
\Gamma_{1}=\exp \left(\frac{g_{0}}{1+\left|u_{1}\right|^{2}}-g_{h}\right)
$$

Here $g_{\mathrm{h}}=1 \mathrm{~dB}$ is amplifier loss at high power limit, and $\left(g_{0}-g_{\mathrm{h}}\right)$ is amplification in opposite limit of low-level signal power. Let's stress that in contrast to previous study ${ }^{8}$, the components of matrix $A_{\Gamma}$ now becomes complex having factor $\exp \left(i \gamma\left|u_{1}\right|^{2}\right)$, what models nonlinear phase shift caused by Kerr nonlinearity inside optical gain segments.

Let's also note that in order to describe the whole system, the matrices of fiber optical elements should be multiplied in the reverse order since the most conventional way is to write vectors to the right of matrices so that the last matrix in the product transforms the vector first and the first matrix in the product acts the last. This yields the matrix $A$ of entire fiber optical system: 


$$
A=A_{C C} \times A_{O C} \times A_{\varphi} \times A_{O C} \times A_{\Gamma} .
$$

The matrix $A$ (3) describes cavity round-trip evolution of optical field amplitudes $\left(u_{1}, u_{2}\right)$ at the beginning of gain/loss segments of fiber cavity shown in Fig. 1.

\section{PT-SYMMETRY IN THE PRESENCE OF KERR NONLINEARITY}

Let's consider influence of Kerr nonlinearity on PT-transition in coupled fiber cavities schematically shown in Fig. 1 using discrete stationary matrix model discussed above. In order to do this let's examine dependencies of intra-cavity power at the middle of gain $\left(P_{\text {gain }}\right)$ and loss $\left(P_{\text {loss }}\right)$ cavities on gain $\mathrm{g}_{0}$ at different levels of nonlinearity $\gamma$ in Eq. (2). In particular, Fig. 2 shows such power dependencies for the case $\gamma=0$ (linear case, see Fig. 2 (a) and (b)), $\gamma=0.01$ for Fig. 2 (c) and (d) and $\gamma=0.1$ for Fig. 2 (e) and (f) respectively. For each case there are two plots with different relative phase shifts $\Delta \varphi=1.5$ and 0.8 for left and right panels column in Fig. 2 respectively.

In the linear case $(\gamma=0)$ relative phase shift $\Delta \varphi=1.5$ provides quite strong cavity coupling what yields PT-symmetric lasing regime. Thus, as can be seen in Fig. 2 (a), powers inside gain and loss cavities coincide for any gain value $\mathrm{g}_{0}$. When gain $\mathrm{g}_{0}$ is less than 0.9 , lasing powers are equal to zero and no lasing occurs. Once gain $\mathrm{g}_{0}$ is above lasing threshold, the powers inside gain and loss cavities grow simultaneously. Another situation can be seen for relatively weak cavities coupling realized at $\Delta \varphi=0.8$, see Fig. 2 (b). Due to weaker cavities coupling, a lower fraction of energy goes through cavity with losses what makes lasing threshold lower, $\mathrm{g}_{0}=0.48 v \mathrm{~s} . \mathrm{g}_{0}=0.9$ for $\Delta \varphi=1.5$ shown in Fig. 2 (a). Once gain $g_{0}$ is above lasing threshold, the power inside gain cavity is almost three times as high as the power inside loss cavity what indicates PT-broken lasing regime.

Now let's see how this picture is changed when a small $(\gamma=0.01)$ Kerr nonlinearity is introduced. It's obvious that such a weak perturbation can result only in a small change of generated powers. Indeed, Fig. 2 (d) looks almost identical to PT-broken lasing shown in Fig. 2 (b). In contrast, in PT-symmetrical case even a small change of powers leads to qualitatively different situation. As could be seen from Fig. 2 (c), the presence of such a small Kerr nonlinearity makes powers inside gain and loss cavities slightly different thus violating exact PT-symmetry what becomes apparent at relatively high power levels realized at relatively large gain $\mathrm{g}_{0}$, see Fig. 2 (c).

Finally, when Kerr nonlinearity is increased up to $\gamma=0.1$, distortion of power dependencies becomes visible even in the case of relatively weak cavities coupling at $\Delta \varphi=0.8$, see Fig. 2 (f). In the case of strong coupling $(\Delta \varphi=1.5)$, powers in the gain and loss cavities evolve in different ways except relatively narrow area close to lasing threshold, see Fig. 2 (e). Let's also note that Fig. 2 (e) and Fig. 2 (f) look quite similar, especially high enough above lasing threshold. Thus we can conclude that Kerr nonlinearity breaks PT-symmetry in such fiber laser system and should be avoided in order to make PT-symmetry observable.

In the case of weak enough Kerr nonlinearity (see, e.g. Fig. 2 (c,d)), PT-symmetry is broken in the strict sense. However from the point of view of experimental measurements where some inaccuracy is inevitable the effect of weak nonlinearity may be negligible providing that the powers are relatively low (e.g. the laser is operated near threshold.) Thus, for example, if we can measure the ratio of powers $P_{\text {loss }} / P_{\text {gain }}$ in the middle of cavities with accuracy of 5\%, we should consider points where $P_{\text {loss }} / P_{\text {gain }}>0.95$ as PT-symmetric area. The results of our simulations suggest that nonlinear phase shift (i.e. product of nonlinear coefficient $\gamma$ and power $\left|u_{1}\right|^{2}$ ) must be less than 0.15 in order to obtain width of PT-symmetric area at the level of $20 \%$. Let's stress that this result is valid for different values of nonlinear coefficient $\gamma$ : the more is $\gamma$ the less should be power $\left|u_{1}\right|^{2}$, so that nonlinear phase shift $\gamma \times\left|u_{1}\right|^{2}$ is less than 0.15 .

\section{NLSE-BASED MODEL}

Considered above stationary matrix model takes into account neither fast temporal dynamics of laser radiation nor its optical spectrum. Let's consider more complex and realistic physical model based on nonlinear Schrödinger equation (NLSE), which has been successfully adopted earlier to simulate spectral, temporal and statistical properties of radiation generated by fiber Raman lasers ${ }^{9-12}$. To simulate propagation of laser radiation inside gain / loss cavities, we used following equation (NLSE): 

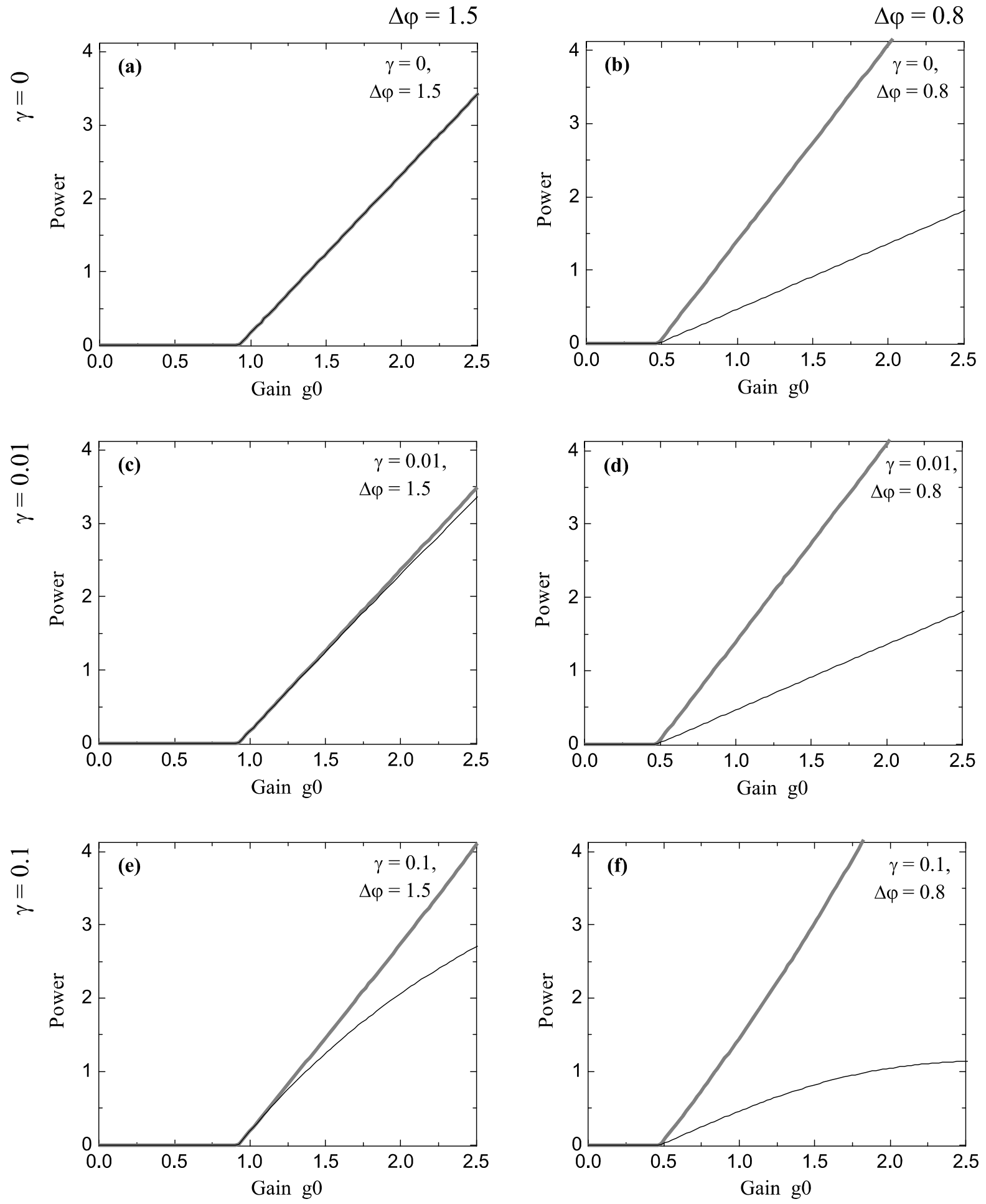

Fig. 2. Dependence of lasing power in two cavities $v s$. gain $\mathrm{g}_{0}$ in PT-symmetric and PT-broken regimes corresponding to different relative phase shift $\Delta \varphi=1.5(\mathrm{a}, \mathrm{c}, \mathrm{e})$ and $\Delta \varphi=0.8(\mathrm{~b}, \mathrm{~d}, \mathrm{f})$ and different nonlinear coefficients $\gamma=0(\mathrm{a}, \mathrm{b}), \gamma=0.01$ $(\mathrm{c}, \mathrm{d})$ and $\gamma=0.1(\mathrm{e}, \mathrm{f})$. 


$$
\frac{\partial A_{s}}{\partial z}+\frac{\alpha_{s}}{2} A_{s}=-\frac{i}{2} \beta_{2 s} \frac{\partial^{2} A_{s}}{\partial t^{2}}+i \gamma_{s}\left|A_{s}\right|^{2} A_{s}+\frac{g_{s}}{2} P_{p} A_{s}
$$

where $A$ stands for field envelope, indices "s" and "p" denote Stokes wave (generated radiation of Raman laser) and optical pumping respectively, $z$ is the longitudinal coordinate along the fibre of length $\mathrm{L}=770 \mathrm{~m}, t$ is the time in the retarded frame of reference co-propagating with Stokes wave, $\gamma=3.5 \times 10^{-3}(\mathrm{~m} \times \mathrm{W})^{-1}$ and $\beta_{2}=5.6 \times 10^{-26} \mathrm{~s}^{2} / \mathrm{m}$ are nonlinear and dispersion coefficients correspondingly, $g_{\mathrm{s}}=6 \times 10^{-4}(\mathrm{~m} \times \mathrm{W})^{-1}$ is Raman coefficient, $\alpha=0.05 \mathrm{~dB} / \mathrm{m}$ stands for linear loss coefficient, $P=|A|^{2}$ is the power. Analogous equation can be written for pump wave ${ }^{9}$. However, since the equations for Stokes wave and pumping are coupled only via powers (not field amplitudes), its more efficient to use much simple ordinary differential equation for $P_{\mathrm{p}}=\left|A_{\mathrm{p}}\right|^{2}$ which can be easily derived from analogue of Eq. (4) for pump wave:

$$
\frac{d P_{p}}{d z}=-\frac{\omega_{p}}{\omega_{s}} g_{s} P_{p} P_{s}-\alpha_{p} P_{p}
$$

Here $\omega_{\mathrm{p}, \mathrm{s}}$ are carrier optical frequencies of pump and Stokes wave respectively. Let's stress that Eq. (4) and (5) are used to simulate evolution of laser radiation and optical pumping inside gain and loss cavities what generalizes the action of matrix $A_{\Gamma}$ within stationary matrix model discussed above. In other words, within NLSE-based model, the matrix elements of $A_{\Gamma}$ are operators according to Eq. (4). These operators transform time-dependent complex amplitudes inside gain and loss cavities instead of stationary field amplitudes as before. All other matrices in the product (3) are the same as earlier. The results of PT transition simulation obtained within NLSE-based model are similar to those in stationary matrix model, see Fig. 3. Left column (Fig. 3 (a), (c) and (e)) corresponds to NLSE-based model, whereas right column (Fig. 3 (b), (d) and (f)) corresponds to stationary matrix model. The upper graphs (Fig. 3 (a) and (b)) correspond to the case of relatively high power lasing (well above lasing threshold.) It could be seen that both models predict distorted powers profile with the only point $\Delta \varphi=\pi$ (or $-\pi$, what's the same) with equal powers inside loss and gain cavities. In contrast, at the point slightly above PT-lasing threshold (Fig. 3 (c) and (d)) one can see that both models predict two distinct areas, one is PT-symmetric lasing with equal powers inside loss and gain cavities, another one is PT-broken area, with $\mathrm{P}_{\text {loss }}<\mathrm{P}_{\text {gain }}$. Finally, at lower pump powers under PT-lasing threshold (Fig. 3 (e) and (f)) both models predict only PT-broken lasing with $\mathrm{P}_{\text {loss }}<\mathrm{P}_{\text {gain }}$. If gain and loss cavities are coupled strong enough (what takes place when relative phase shift $\Delta \varphi$ is large enough), no lasing occurs what corresponds to the presence of solution in Fig. 3 (e,f) only in some area around $\Delta \varphi=0$ bounded with dashed lines.

\section{CONCLUSION}

Thus we investigated a concept of coupled fiber loops with Raman gain and losses exhibiting PT-symmetry. Adjustable phase shift between fiber loops allows one to observe a PT-transition between PT-symmetric and PT-broken lasing states. By changing the phase shift or/and amplification (loss) in fiber loops, one can switch between generation regimes, realizing either PT-symmetric or PT-broken solution. In the PT-symmetric lasing regime, equal powers are generated in both cavities despite only active one is pumped. Using discrete stationary matrix model and NLSE-based model we demonstrated how PT-transition is affected by Kerr nonlinearity. In particular we showed absence of "exact" PTsymmetry in the case when nonlinear phase shift is present, and demonstrated "approximate" PT-symmetry. The bound between areas of different symmetry becomes diffused and vanishes at high enough nonlinear phase shifts both in stationary and NLSE-based models.

\section{ACKNOWLEDGMENTS}

This work was supported by the Russian Science Foundation (16-12-10402). A.A.S. acknowledges support by the Australian Research Council (DP160100619). 

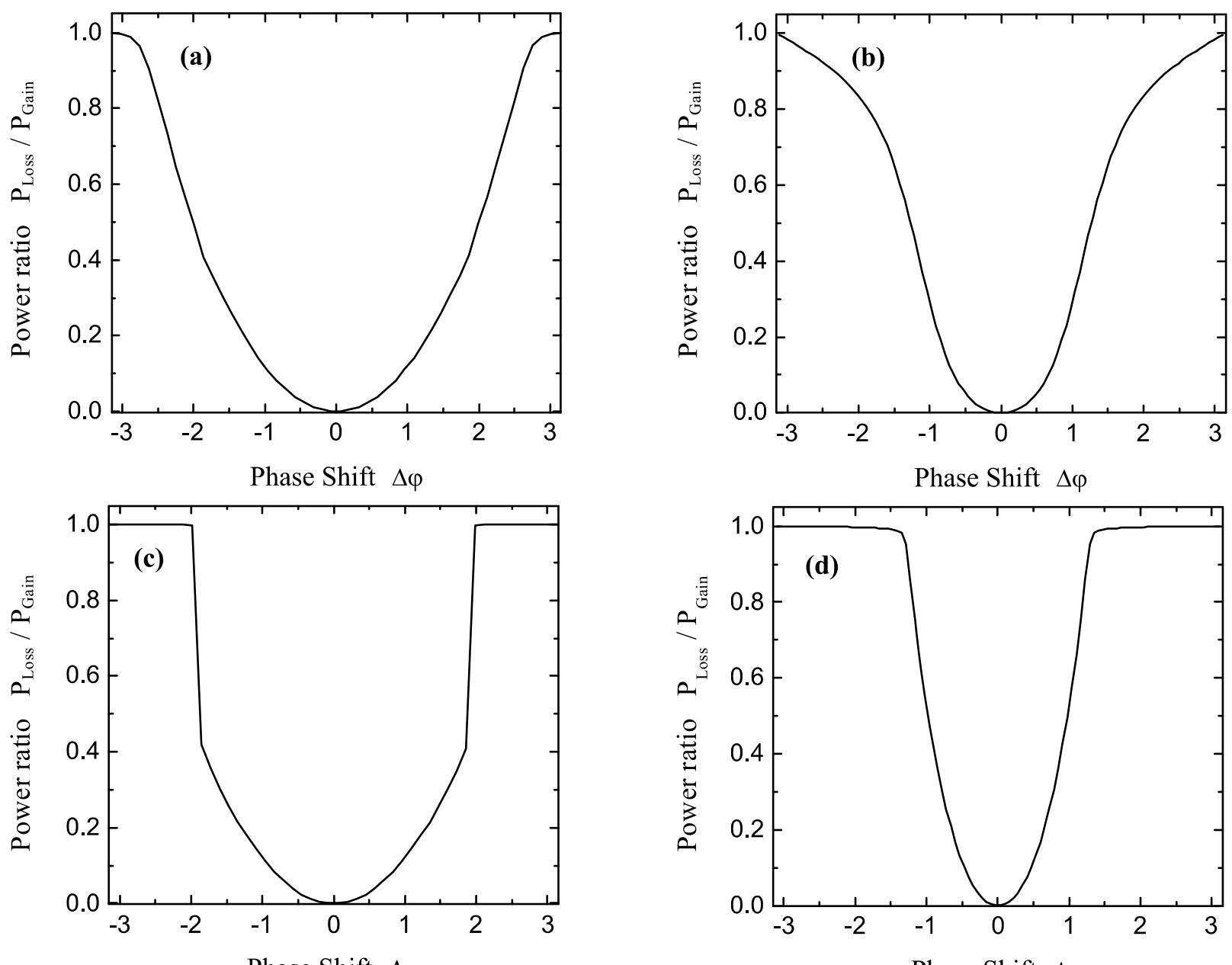

Phase Shift $\Delta \varphi$
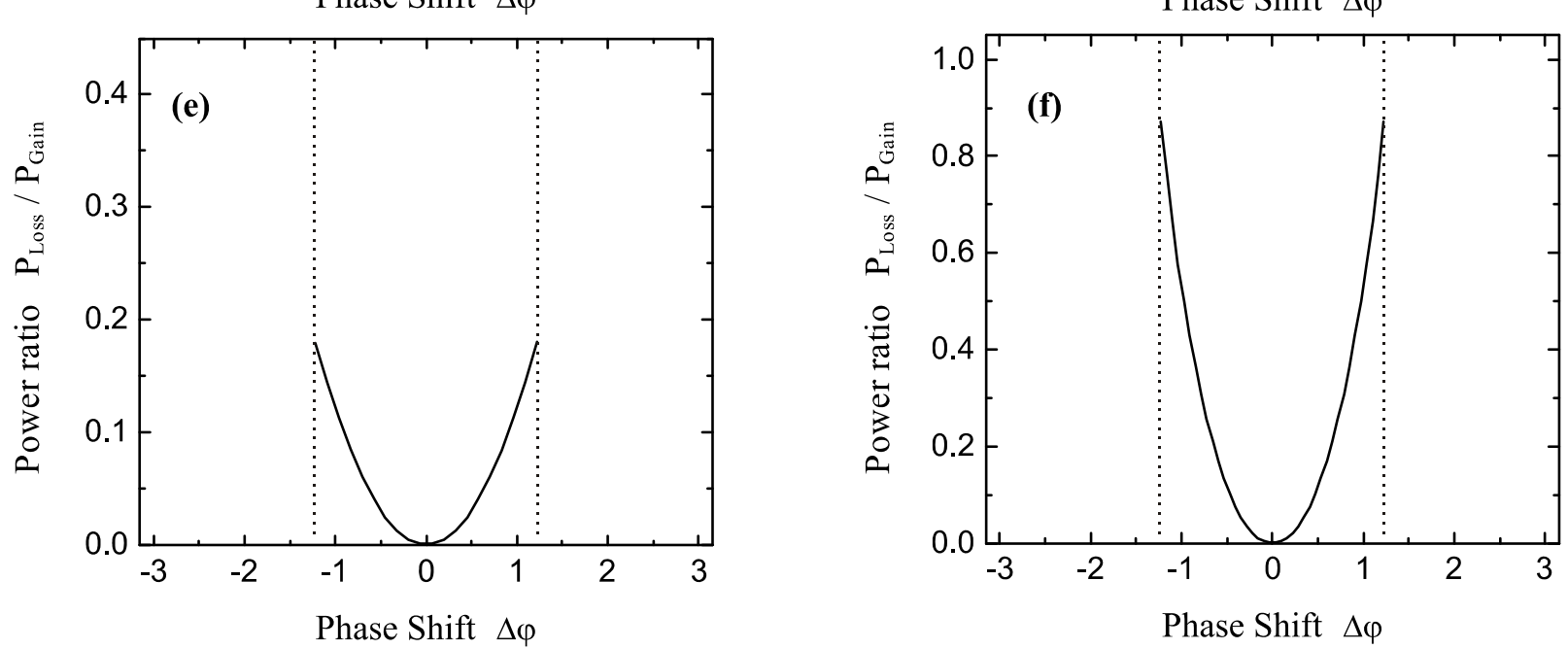

Fig. 3. Ratio of intra-cavity powers Ploss /Pgain $v$ s. relative phase shift $\Delta \varphi$ obtained within NLSE-based model at pump power level (a) $\mathrm{P}=4 \mathrm{~W}$, (b) $3.082 \mathrm{~W}$, (e) $3.020 \mathrm{~W}$ and within stationary matrix model at (b) $\mathrm{g}_{0}=2.5$, (d) $\mathrm{g}_{0}=0.95$ and (f) $\mathrm{g}_{0}=0.90$. 


\section{REFERENCES}

[1] Feng, L., Wong, Z. J., Ma, R. M., Wang, Y. and Zhang, X., "Single-mode laser by parity-time symmetry breaking," Science 346 (6212), 972-975 (2014).

[2] Hodaei, H., Miri, M. A., Heinrich, M., Christodoulides, D. N. and Khajavikhan, M., "Parity-time-symmetric microring lasers," Science 346 (6212), 975-978 (2014).

[3] Hodaei, H., Miri, M. A., Hassan, A. U., Hayenga, W. E., Heinrich, M., Christodoulides, D. N. and Khajavikhan, M., "Single mode lasing in transversely multi-moded PT-symmetric microring resonators," Laser Photon. Rev. 10 (3), 494-499 (2016).

[4] Brandstetter, M., Liertzer, M., Deutsch, C., Klang, P., Schoberl, J., Tureci, H. E., Strasser, G., Unterrainer, K. and Rotter, S., "Reversing the pump dependence of a laser at an exceptional point," Nat. Commun. 5, 4034 (2014).

[5] Longhi, S. "PT -symmetric mode-locking," Opt. Lett. 41(19), 4518-4521 (2016).

[6] Jahromi, A. K., Hassan, A. U., Abouraddy, A. and Christodoulides, D., "Robust statistical parity-time symmetric lasers in fiber cavities," Frontiers in Optics, JW4A.100 (2016).

[7] Smirnov, S. V., Churkin, D. V., Makarenko, M., Vatnik, I., Suchkov, S. V., Sukhorukov, A. A., "PT-symmetry of coupled fiber lasers", Proc. SPIE 10457, AOPC 2017: Laser Components, Systems, and Applications, 104572Y (2017).

[8] Smirnov, S. V., Makarenko M. O., Suchkov, S. V., Churkin, D. V., Sukhorukov, A. A., "Bistable lasing in parity-time symmetric coupled fiber rings," Photonics Research 6 (4), A18-A22 (2018).

[9] Churkin, D. V., Smirnov, S. V., Podivilov, E. V., "Statistical properties of partially coherent cw fiber lasers," Opt. Lett. 35 (19), 3288-3290 (2010).

[10] Churkin, D. V., Gorbunov, O. A., Smirnov, S. V., "Extreme value statistics in Raman fiber lasers,” Opt. Lett. 36 (18), 3617-3619 (2011).

[11]Churkin, D. V., Smirnov, S. V., "Numerical modeling of spectral, temporal and statistical properties of Raman fiber lasers," Opt. Comm. 285 (8), 2154-2160 (2012).

[12] Smirnov, S. V., Tarasov, N., Churkin, D. V., "Radiation build-up in laminar and turbulent regimes in quasi-CW Raman fiber laser," Opt. Express 23 (21), 27606-27611 (2015). 